\title{
Detection of Malignancy in Digital Mammograms from Segmented Breast Region Using Morphological Techniques
}

\author{
Prakash Bethapudi Member IEEE ${ }^{1}$, Dr. E. Srinivasa Reddy , Dr.Madhuri.P ${ }^{3}$ \\ ${ }^{I}$ (Assistant Professor, Computer Science and Engineering, GIT, GITAM UNIVERSITY, INDIA) \\ ${ }_{2}^{2}$ (Professor, Computer Science and Engineering, ACHARYA NAGARJUNA UNIVERSITY, INDIA) \\ ${ }^{3}$ (Consultant Radiologist, Mahatma Gandhi Cancer Hospital, Visakhapatnam, A.P, INDIA)
}

\begin{abstract}
Mammography is an efficient and contemporary option in diagnosing breast cancer among all ages of women. Nevertheless, the radiologist's has remarkable influence on revelation of the mammogram. It is a difficult and challenging task in identifying the masses in the breast region of a digital mammography. The proposed research intends to develop an image processing algorithm in identifying malignancy by using an automated segmentation technique for mammogram. The proposed work deals with an approach for extracting the malignant masses in mammograms for detection of breast cancer. The work proposed is based on the following procedure: (a)Removing the noise and the background information. (b)Applying thresholding and retrieving the largest region of interest (ROI). (c)Performing the morphological operations and extracting the ROI and identifying the malignant mass from the screened images of the breast. This method was tested over several images of various patients taken from a cancer hospital and implemented using Matlab code. Thus, capable in executing the pre-processed image effectively and detected the segmentation region and identified the malignant data for assessment.
\end{abstract}

Keywords - Malignancy, Mammogram, Morphological, ROI, Segmentation, Thresholding.

\section{INTRODUCTION}

Breast cancer is the most common form of cancer identified often in most of the women and is the major cause of women mortality [1]. The Mortality rate can be decreased by increasing the number of cancers being diagnosed. The mortality rate has been reduced remarkably in the past decade due increase in screening programs [2]. Detection of breast cancer increase the survival rate whereas delayed diagnosis frequently brazen out the patient to an unrecoverable stage and hence results in death [3]. Mammography is most efficient, effective and reliable technique currently being used by most of the radiologist's to detect breast cancer at various stages. Computer aided detection and diagnosis are being used by most of the radiologists in detection of breast cancer [4]. Digital mammography uses the DICOM data which is an image where all details of the patient such as Name, Age, Gender, Address, Patient-ID, Date, Size(WL,WW) and frame are present on the image and are included in the object classes. The DICOM formatted images which contains the details of the patient are more important for the radiologists in performing their diagnosis. The features of masses in mammograms greatly vary in their size and shapes. Based on these sizes and shapes the type of cancer Benign or Malignant is identified. The present study is focused on image processing for segmentation of breast and detecting the type of cancer i.e., malignant based on image Enhancement techniques and few morphological operations performed on the screened images. This paper is organized as follows. Section II provides some information about mammogram segmentation. Section III involves the techniques being used in this paper to identify the malignancy in the breast. Section IV contains the experimental results of the techniques that are described in this paper. And the final section $\mathrm{V}$ consists of conclusion and the further work information.

\section{IMAGE SEgMENTATION}

Segmentation is an Image Processing technique that is used in classifying the image into various distinct regions which includes breast border [5], the nipple [6] and the pectoral muscle. Mammographic segmentation is the process of dividing an image into multiple parts. This is typically used to identify objects or other relevant information in digital images. A great variety of segmentation methods has been proposed. There have been various approaches proposed to the task of segmenting the breast profile region in mammograms. Some of these have focused on using threshold [7], gradients [8], modeling of the non-breast region of a mammogram using a polynomial or active contours [9]. The following are few categories from available techniques.

Threshold based segmentation: Here Histogram thresholding and slicing techniques are used to segment the image. They may be applied directly to an image, but can also be combined with pre- and post-processing techniques. 
- Edge based segmentation: With this technique, detected edges in an image are assumed to represent object boundaries, and are used to identify these objects.

- Region based segmentation: An edge based technique may attempt to find the object boundaries and then locate the object itself by filling them in, But a region based technique takes the opposite approach, by (e.g.) starting in the middle of an object and then "growing" outward until it meets the object boundaries.

- Clustering techniques: Although clustering is sometimes used as a synonym for (agglomerative) segmentation techniques, we use it here to denote techniques that are primarily used in exploratory data analysis of high-dimensional measurement patterns. In this context, clustering methods attempt to group together patterns that are similar in some sense. This goal is very similar to what we are attempting to do when we segment an image, and indeed some clustering techniques can readily be applied for image segmentation.

- Matching: When we know what an object we wish to identify in an image (approximately) looks like, we can use this knowledge to locate the object in an image. This approach to segmentation is called matching.

The image segmentation technique that is used in this paper is Threshold based image segmentation. Thresholding is probably the most frequently used technique to segment an image. The thresholding operation is a grey value remapping operation $g$ defined by:

$$
\mathrm{g}(v)=\left\{\begin{array}{l}
0 \text { if } v<t \\
1 \text { if } v \geq t
\end{array}\right.
$$

Where $v$ represents a grey value and $t$ is the threshold value. Thresholding maps a grey-valued image to a binary image. After the thresholding operation, the image has been segmented into two segments, identified by the pixel values 0 and 1 respectively. Before starting the actual process, first convert the DICOM imageFig.1 (a) to ".jpg" image Fig.1 (b). Remove the details such as name, age, gender, address, size, frame etc,. Those are available on the screened image Fig.1 (a) and then start implementation on the image Fig.1 (b).

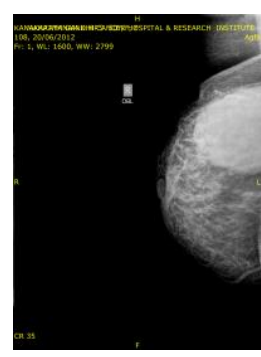

(a)

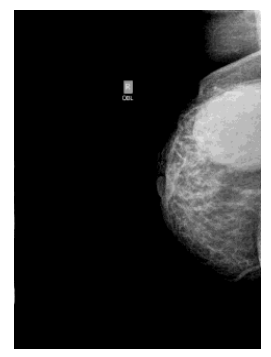

(b)

Fig1. Converting DICOM image (a) to .jpg image (b) by removing all the details that are available on the image

\section{Noise Removal}

\section{TECHNIQUES USED}

Majority of acquired mammogram images consisting of noise which are visible and invisible are filtered by using Gaussian Filtering approach. Gaussian filters are a class of linear smoothing filters with the weights chosen according to the shape of a Gaussian function. The Gaussian smoothing filter is a very good filter for removing noise drawn from a normal distribution! Gaussian functions have five properties that make them particularly useful in early vision processing. These properties indicate that the Gaussian smoothing filters are effective low-pass filters from the perspective of both the spatial and frequency domains, are efficient to implement, and can be used effectively in practical vision applications.

\section{Image Enhancement}

Image enhancement is the process of adjusting digital images so that the results are more suitable for display or further analysis. We remove noise or brighten an image, making it easier to identify key features. During image acquisition such as scanner-induced artifacts, excessive background noise, scratches and dust artifacts could influence the reliability of this algorithm. The mammogram presented in Fig.2 (a) has a highly non-uniform background and very little contrast in the area above the core breast tissue region. So image enhancement is required before segmentation and brightness is added to the image Fig.2 (b). 


\section{Remove Background information}

In order to remove the background information such as wedges and labels in the mammogram images, first convert the grayscale or colour image to binary image by using threshold technique and morphological operations. Thresholding is the simplest method of image segmentation. From a grayscale image, thresholding can be used to create binary images. Fig. 2(c) shows a mammogram image after apply simple thresholding at level 0 . After the gray scale mammogram images are converted into binary, remove the noise or background information from the binary image by removing all the smaller objects except the largest mammography part Fig.2 (d). The algorithm steps to find artefacts and labels and to separate breast profile are as follows:

- Convert the mammogram into binary using threshold technique.

- Binary image objects are labelled and number of pixels in all objects is counted.

- All binary objects are cleaned except the largest one: breast profile (Fig. 2(d)). After which the morphological operation to remove isolated pixels is applied.

- The resulting binary image is multiplied with the original mammogram image to get the final image without artefacts.

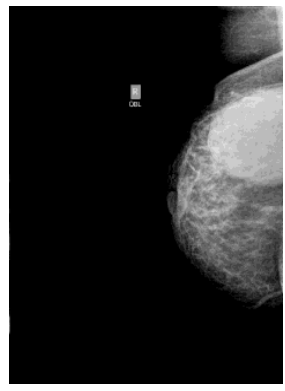

a

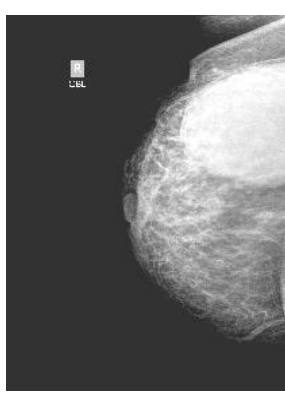

b

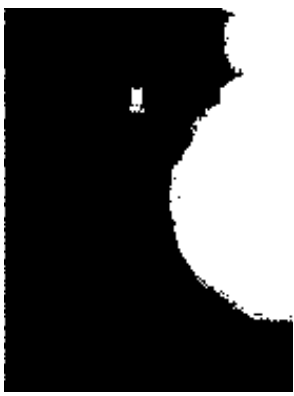

c

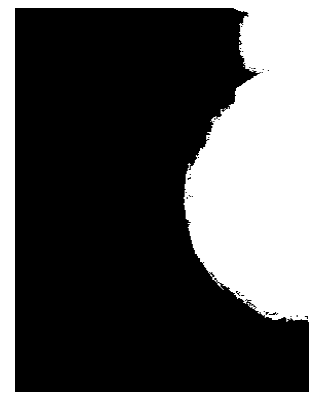

d

Fig.2 Image after applying Gaussian filter (a) and after adding brightness (b) image after applying thresholding $(\mathrm{c})$ and image after eliminating noise and selecting the largest mammographic region (d)

\section{Perform Subtraction}

Image subtraction or pixel subtraction is a process whereby the digital numeric value of one pixel or whole image is subtracted from another image which makes the background disappear leaving only the target. It simply compares the previous frame image with the current one. Image subtraction [10], is a tool for transient object discovery and characterization, $\mathrm{Z}=\operatorname{imsubtract}(\mathrm{X}, \mathrm{Y})$ subtracts each element in array $\mathrm{Y}$ from the corresponding element in array $\mathrm{X}$ and returns the difference in the corresponding element of the output array $\mathrm{Z}$. $\mathrm{X}$ and $\mathrm{Y}$ are real, non sparse numeric arrays of the same size and class, or $\mathrm{Y}$ is a double scalar. The array returned, $\mathrm{Z}$, has the same size and class as $\mathrm{X}$ unless $\mathrm{X}$ is logical, in which case $\mathrm{Z}$ is double. If $\mathrm{X}$ is an integer array, elements of the output that exceed the range of the integer type are truncated, and fractional values are rounded. In this paper I implemented subtraction on two images, the segmented image Fig.2 (d) and the converted RGB image Fig.3 (a) and finally obtained the tumor that is present in the screened mammographic image.

\section{Perform Erosion}

Erosion is one of two fundamental operations in morphological image processing from which all other morphological operations are based. It was originally defined for binary images, later being extended to grayscale images, and subsequently to complete lattices. The erosion [11], of $\mathrm{f}$ by a flat structuring element $\mathrm{b}$ at any location $(\mathrm{x}, \mathrm{y})$ is defined as the minimum value of the image in the region coincident with $\mathrm{b}$ when the origin of $\mathrm{b}$ is at $(\mathrm{x}, \mathrm{y})$. Therefore, the erosion at $(\mathrm{x}, \mathrm{y})$ of an image $\mathrm{f}$ by a structuring element $\mathrm{b}$ is given by:

$$
f \ominus b(x, y)=\min \{f \mid(x+s, y+t)\}
$$

where, similarly to the correlation, $x$ and $y$ are incremented through all values required so that the origin of $b$ visits every pixel in $f$. That is, to find the erosion of $f$ by $b$, we place the origin of the structuring element at every pixel location in the image. The erosion is the minimum value of $f$ from all values of $f$ in the region of $f$ coincident with $\mathrm{b}$. Erosion of a binary image $\mathrm{f}$ by a structuring element $\mathrm{s}$ (denoted $\mathrm{f} \Theta_{\mathrm{s}}$ ) produces a new binary image $\mathrm{g}=\mathrm{f} \Theta_{\mathrm{s}}$ with ones in all locations $(\mathrm{x}, \mathrm{y})$ of a structuring element's origin at which that structuring element $\mathrm{s}$ fits the input image f, i.e. $\mathrm{g}(\mathrm{x}, \mathrm{y})=1$ is $\mathrm{s}$ fits $\mathrm{f}$ and 0 otherwise, repeating for all pixel coordinates $(\mathrm{x}, \mathrm{y})$. 
Erosion removes small-scale details from a binary image but simultaneously reduces the size of regions of interest, too.

\section{EXPERIMENTAL RESULTS}

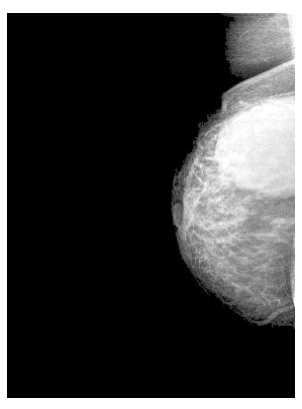

(a)

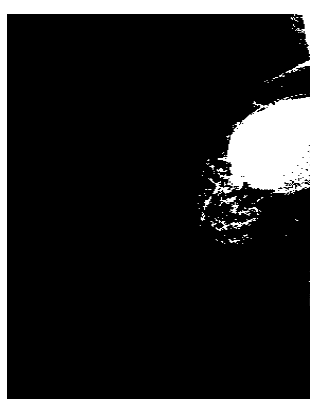

(b)

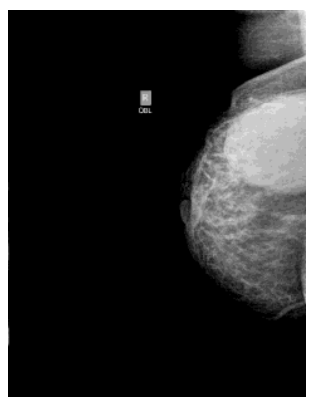

(c)

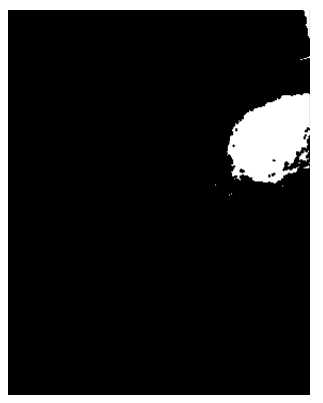

(d)

Fig. 3 The Resultant mammogram without any artifacts (a) and the result after performing image subtraction (b) Original Image(c), The resultant image after applying Erosion (d)

\section{CONCLUSION AND FUTURE WORKS}

Identifying the breast cancer is a challenging problem in medical image processing and medical field. Digital mammograms on a particular segmentation algorithm make it difficult to identify breast cancer accurately. The acquisition parameters also influence the quality of the image. Mammography segmentation using techniques presented in this paper is efficient in getting the malignant breast cancer region, which help the doctors to concentrate more on that particular region for examination and treatment. For the future work it may be planned to develop an algorithm to acquire a smoother breast region for image pre-processing, study the behavior of the breast, improve the edge detection and region segmentation algorithms which detect the abnormalities in segmented images and produce more accurate results than existing methods for the detection of breast cancer.

\section{REFERENCES}

[1]. R.A. Smith, "Epidemiology of breast cancer in a categorical course in physics," Technical Aspects of Breast Imaging, 2nd ed., RSNA publication, Oak Book, II, pp.21, 1993..

[2]. R. Peto, J. Boreham, M. Clarke, C. Davies., V. Beral, "UK and USA Breast cancer deaths down $25 \%$ in year 2000 at ages $20-69$ years", THE LANCET, Volume 355, Issue 9217, Page 1822, 20 May 2000.

[3]. Ranadhir Ghosh, Moumita Ghosh, John Yearwood, "A Modular Framework for Multi category feature selection in Digital mammography", In Proceedings of the $12^{\text {th }}$ European Symposium On Artificial Neural Networks ESANN'2004, Bruges (Belgium), pp. 175-180, 28-30 April 2004.

[4]. Armen Sahakyan, Hakop Sarukhanyan,"Segmentation of the Breast Region in Digital Mammograms and Detection of Masses",IJACSA,Volume.3,No.2,2012.

[5]. R.Chandrasekhar, and Y. Attikiouzel, "Automatic Breast Border Segmentation by Background Modelling and Subtraction”, in 5th International Workshop on Digital Mammography (IWDM), (Yaffe M. ed.), Medical Physics Publishing, Madison, USA, pp. 560565 .

[6]. R.Chandrasekhar, and Y. Attikiouzel, "A Simple Method for Automatically Locating the Nipple on Mammograms", IEEE Transaction on Medical Imaging, Vol. 16, pp.483-494,Oct. 1997

[7]. U.Bick,M.L.Giger,R.A.Schmidt,R.M.Nishikawa,D.E,Wolverton and K.Doi, “Automated Segmentation of Digitized Mammograms,Academic Radiology, Vol.2,no.2,pp.1-9,1995

[8]. A.J. Mendez, P.J. Tahoces, M.J. Lado, M. Souto, J.L. Correa, and J.J. Vidal, J.J, “Automatic Detection of Breast Border and Nipple in Digital Mammograms", Computer Methods and Programs in Biomedicine, vol. 49, pp. 253-262, 1996.

[9]. M. A. Wirth, A. Stapinski, "Segmentation of the breast region in mammograms using active contours", in Visual Communications and Image Processing, pp.1995-2006.

[10]. R.Chandrasekhar,Y.Attikiouzel,“Breast border Segmentation by Background Modeling and Subtraction”M.J.Yaffe(Ed), Proceedings of $5^{\text {th }}$ (IWDM) International Workshop on Digital Mammography,Medical Physics Publishing, Toronto, Canada, 2000,pp.560-565.

[11]. Yao Yao, "Segmentation of Breast Cancer in Mammograms and Detection using Magnetic Resonance Imaging" 